\title{
Near-Field Localization of Acoustic Sources with Imperfect Spatial Coherence, Distributed Processing, and Low Communication Bandwidth
}

\author{
Richard J. Kozick ${ }^{a}$ and Brian M. Sadler ${ }^{b}$ \\ ${ }^{a}$ Bucknell University, Dept. of Electrical Engineering, Lewisburg, PA 17837 \\ ${ }^{b}$ Army Research Laboratory, AMSRL-CI-CN, 2800 Powder Mill Road, Adelphi, MD 20783
}

\begin{abstract}
Multiple sensor arrays distributed over a region provide the means for accurate localization of the $(x, y)$ position of a source. When microphone arrays are used to measure aeroacoustic signals from ground vehicles, random fluctuations in the air lead to frequency-selective coherence of the signals that arrive at widely-separated arrays. We have shown previously that even in cases of imperfect spatial coherence, improvements in source localization accuracy are possible when the data from widely-separated arrays are processed jointly by a fusion center. Further, we have shown that a distributed processing scheme involving bearing estimation at individual arrays and time-delay estimation (TDE) between pairs of widely-separated sensors performs nearly as well as the optimum scheme, with significantly lower communication bandwidth. These results were obtained by studying the Cramér-Rao bound (CRB) on source localization accuracy based on a statistical model for the data measured at the sensors. Refined bounds (Ziv-Zakai) are presented in this paper that imply a threshold value of coherence is needed to achieve accurate TDE between widely-separated sensors. The threshold coherence is a function of the signal to noise ratio, fractional bandwidth, and time-bandwidth product of the observed signals. Results are presented from measured aeroacoustic data that illustrate TDE with widely-separated sensors.
\end{abstract}

Keywords: aeroacoustic sensor arrays, imperfect spatial coherence, Ziv-Zakai bounds, time-delay estimation, nearfield source localization.

\section{INTRODUCTION}

We are concerned with estimating the location $\left(x_{s}, y_{s}\right)$ of a wideband source using multiple sensor arrays that are distributed over an area. We consider schemes that distribute the processing between the individual arrays and a fusion center in order to limit the communication bandwidth between arrays and fusion center. Triangulation is a standard approach for source localization with multiple sensor arrays. Each array estimates a bearing and transmits the bearing to the fusion center, which combines the bearings to estimate the source location $\left(x_{s}, y_{s}\right)$. Triangulation is characterized by low communication bandwidth and low complexity, but it ignores coherence that may be present in the wavefronts that are received at distributed arrays. In this paper, we investigate new approaches for source localization with multiple arrays that exploit partial coherence of the wavefronts at distributed arrays. We have shown previously ${ }^{1}$ that the Cramér-Rao lower bound (CRB) on the variance of source location estimates is reduced when coherence from array to array is exploited. We also showed ${ }^{1}$ that the CRB changes little for suboptimum source localization methods that employ distributed processing to reduce the communication bandwidth between the arrays and the fusion center.

We focus on the case of narrowband processing in this paper. In particular, we investigate time delay estimation (TDE) with partially-coherent signals received by widely-separated sensor arrays. We showed ${ }^{1}$ that combining the TDEs with bearing estimates from the individual arrays has nearly the same source localization CRB as the optimum method that jointly processes of all of the sensor data. However, bearing estimation with TDE requires much less communication bandwidth than the optimum method. We have described elsewhere ${ }^{2}$ a subspace algorithm for processing partially-coherent signals received by widely-separated sensor arrays.

We model the signals measured at the distributed sensor arrays as jointly Gaussian wideband random processes. The model accounts for propagation effects between the source and the distributed arrays, including frequencyselective spatial coherence and different signal power spectra received at each array. The spatial coherence of the

Author email: kozick@bucknell.edu and bsadler@arl.army.mil 
wavefronts is modeled as perfect over each individual array but imperfect between distinct arrays. This idealization allows us to study the effect of varying coherence between arrays on source localization accuracy. Physical modeling of frequency-selective coherence is discussed by Wilson. ${ }^{3}$ The power spectral density of the source is arbitrary, allowing a range of cases to be modeled, including narrowband sources, sums of harmonics, and wideband sources with continuous power spectra.

Previous work on source localization with acoustical arrays has focused on angle of arrival estimation with a single array. ${ }^{4-7}$ These works use the coherent wideband focusing approach ${ }^{8,9}$ to combine correlation matrices from different narrowband frequency bins into a single correlation matrix that admits subspace processing. The problem of imperfect spatial coherence in the context of narrowband angle-of-arrival estimation with a single array has been studied. ${ }^{10-13}$ Paulraj and Kailath ${ }^{10}$ presented a MUSIC algorithm that incorporates the nonideal spatial coherence, assuming that the coherence variation is known. Gershman et al. ${ }^{11}$ provided a procedure to jointly estimate the spatial coherence loss and the angles of arrival. Song and Ritcey ${ }^{12}$ provide maximum-likelihood methods for estimating the parameters of a coherence model and the angles of arrival, and Wilson ${ }^{13}$ incorporates physical models for the spatial coherence. The problem of decentralized array processing has been studied. ${ }^{14,15}$ Wax and Kailath ${ }^{14}$ present subspace algorithms for narrowband signals and distributed arrays, assuming perfect spatial coherence across each array but neglecting the spatial coherence between arrays. Weinstein ${ }^{15}$ presents performance analysis for pairwise processing the wideband sensor signals from a single array and shows negligible loss in localization accuracy when the SNR is high.

The paper is organized as follows. Section 2 describes our model for partially coherent signals observed by the distributed sensor arrays. Also included in Section 2 are results from measured data to illustrate that partial signal coherence is present in measured aeroacoustic data with sensors separated by hundreds of meters. The CRB expressions for source location accuracy are reviewed in Section 3. Section 4 presents fundamental bounds on timedelay estimation that are an extension of the Ziv-Zakai bounds ${ }^{16}$ to the case of partially coherent signals. Examples of processing measured data are included in Section 4, and Section 5 contains a brief discussion of our continuing work.

\section{DATA MODEL}

A model is formulated in this section for the discrete-time signals received by the sensors in distributed arrays. Consider a single source that is located at coordinates $\left(x_{s}, y_{s}\right)$ in the $(x, y)$ plane. Then $H$ arrays are distributed in the same plane, as illustrated in Figure 1. Each array $h \in\{1, \ldots, H\}$ contains $N_{h}$ sensors, and has a reference sensor located at coordinates $\left(x_{h}, y_{h}\right)$. The location of sensor $n \in\left\{1, \ldots, N_{h}\right\}$ is at $\left(x_{h}+\Delta x_{h n}, y_{h}+\Delta y_{h n}\right)$, where $\left(\Delta x_{h n}, \Delta y_{h n}\right)$ is the relative location with respect to the reference sensor. If $c$ is the speed of propagation, then the propagation time from the source to the reference sensor on array $h$ is

$$
\tau_{h}=\frac{r_{h}}{c}=\frac{1}{c}\left[\left(x_{s}-x_{h}\right)^{2}+\left(y_{s}-y_{h}\right)^{2}\right]^{1 / 2} .
$$

We will assume that the wavefronts are well approximated by plane waves over the aperture of individual arrays. Then the propagation time from the source to sensor $n$ on array $h$ will be expressed by $\tau_{h}+\tau_{h n}$, where

$$
\tau_{h n} \approx-\frac{1}{c}\left[\frac{x_{s}-x_{h}}{r_{h}} \Delta x_{h n}+\frac{y_{s}-y_{h}}{r_{h}} \Delta y_{h n}\right]=-\frac{1}{c}\left[\left(\cos \phi_{h}\right) \Delta x_{h n}+\left(\sin \phi_{h}\right) \Delta y_{h n}\right]
$$

where $\tau_{h n}$ is the propagation time from the reference sensor on array $h$ to sensor $n$ on array $h$, and $\phi_{h}$ is the bearing of the source with respect to array $h$. Note that while the far-field approximation (2) is reasonable over individual array apertures, the wavefront curvature that is inherent in (1) must be retained in order to accurately model the (possibly) wide separation between arrays.

The time signal received at sensor $n$ on array $h$ due to the source will be represented as $s_{h}\left(t-\tau_{h}-\tau_{h n}\right)$, where the vector of signals $\mathbf{s}(t)=\left[s_{1}(t), \ldots, s_{H}(t)\right]^{T}$ received at the $H$ arrays are modeled as real-valued, continuous-time, zero-mean, wide-sense stationary, Gaussian random processes with $-\infty<t<\infty$. These processes are fully specified by the $H \times H$ cross-correlation function matrix

$$
\mathbf{R}_{s}(\tau)=E\left\{\mathbf{s}(t+\tau) \mathbf{s}(t)^{T}\right\}
$$




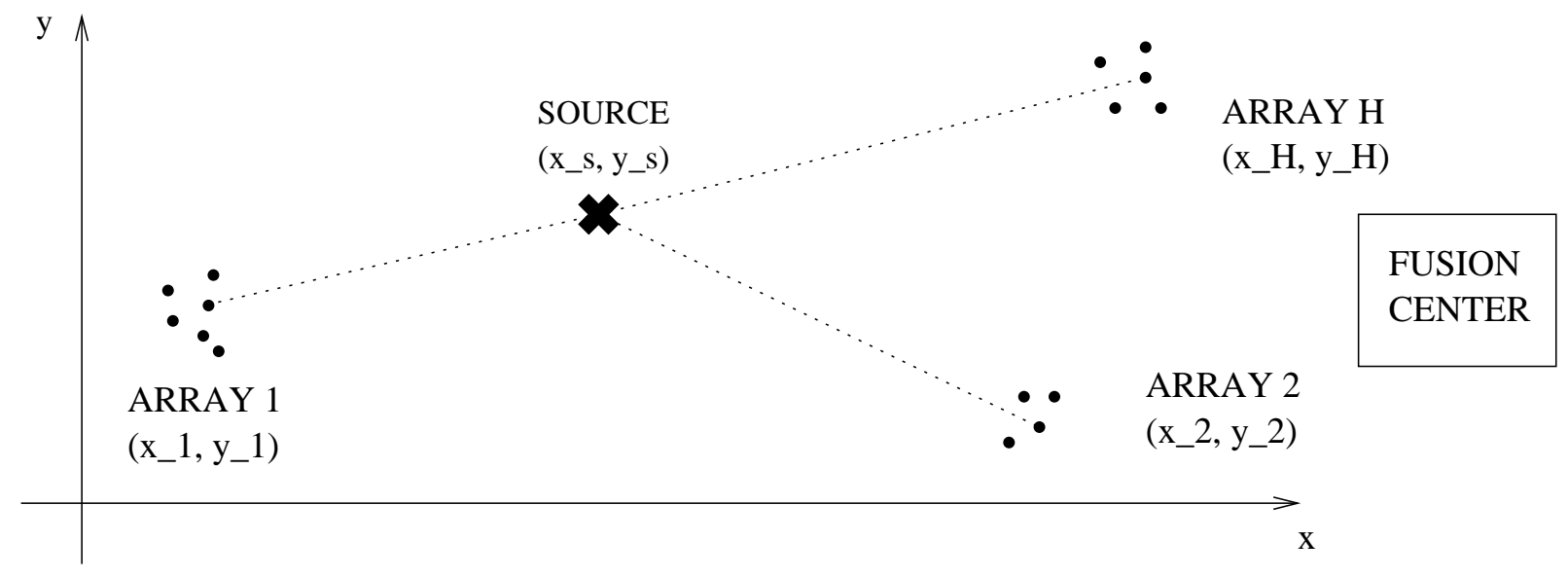

Figure 1. Geometry of source location and $H$ distributed sensor arrays. A communication link is available between each array and the fusion center.

where $E$ denotes expectation, superscript $T$ denotes transpose, and we will later use the notation superscript $*$ and superscript $H$ to denote complex conjugate and conjugate transpose, respectively. The $(g, h)$ element in $(3)$ is the cross-correlation function

$$
r_{s, g h}(\tau)=E\left\{s_{g}(t+\tau) s_{h}(t)\right\}
$$

between the signals received at arrays $g$ and $h$. The correlation functions (3) and (4) are equivalently characterized by their Fourier transforms, which are the cross-spectral density functions

$$
G_{s, g h}(\omega)=\mathcal{F}\left\{r_{s, g h}(\tau)\right\}=\int_{-\infty}^{\infty} r_{s, g h}(\tau) \exp (-j \omega \tau) d \tau
$$

and the associated cross-spectral density matrix

$$
\mathbf{G}_{s}(\omega)=\mathcal{F}\left\{\mathbf{R}_{s}(\tau)\right\}
$$

The diagonal elements $G_{s, h h}(\omega)$ of (6) are the power spectral density (PSD) functions of the signals $s_{h}(t)$, and hence they describe the distribution of average signal power with frequency. The model allows the average signal power to vary from one array to another. Indeed, the PSD may vary from one array to another to reflect propagation differences, source aspect angle differences, and other effects that lead to coherence degradation in the signals at distributed arrays.

Let us elaborate the definition and the meaning of coherence between the signals $s_{g}(t)$ and $s_{h}(t)$ received at distinct arrays $g$ and $h$. In general, the cross-spectral density function (5) can be expressed in the form

$$
G_{s, g h}(\omega)=\gamma_{s, g h}(\omega)\left[G_{s, g g}(\omega) G_{s, h h}(\omega)\right]^{1 / 2},
$$

where $\gamma_{s, g h}(\omega)$ is the spectral coherence function, which has the property $0 \leq\left|\gamma_{s, g h}(\omega)\right| \leq 1$. The coherence function $\gamma_{s, g h}(\omega)$ is generally complex-valued, but we will model it as real-valued. This is a reasonable assumption for acoustic propagation environments in which the loss of coherence is due to random changes in the propagation path length. ${ }^{13,3}$ Note that our assumption of perfect spatial coherence across individual arrays implies that the random changes in the propagation path length have negligible impact on the intra-array delays in (2). Thus the apparent bearing $\phi_{h}$ to array $h$ remains fixed, but the inter-array path delays are randomly perturbed from their deterministic values given by (1). These random effects are modeled by the signal coherence function, $\gamma_{s, g h}(\omega)$.

We model the signal received at sensor $n$ on array $h$ as a sum of the delayed source signal and noise,

$$
z_{h n}(t)=s_{h}\left(t-\tau_{h}-\tau_{h n}\right)+w_{h n}(t),
$$

where the noise signals $w_{h n}(t)$ are modeled as real-valued, continuous-time, zero-mean, wide-sense stationary, Gaussian random processes that are uncorrelated at distinct sensors. That is, the noise correlation properties are

$$
E\left\{w_{g m}(t+\tau) w_{h n}(t)\right\}=r_{w}(\tau) \delta_{g h} \delta_{m n}
$$


where $r_{w}(\tau)$ is the noise autocorrelation function, and the noise power spectral density is $G_{w}(\omega)=\mathcal{F}\left\{r_{w}(\tau)\right\}$. We then collect the observations at each array $h$ into $N_{h} \times 1$ vectors $\mathbf{z}_{h}(t)=\left[z_{h 1}(t), \ldots, z_{h, N_{h}}(t)\right]^{T}$ for $h=1, \ldots, H$, and we further collect the observations from the $H$ arrays into a $\left(N_{1}+\cdots+N_{H}\right) \times 1$ vector

$$
\mathbf{Z}(t)=\left[\begin{array}{c}
\mathbf{z}_{1}(t) \\
\vdots \\
\mathbf{z}_{H}(t)
\end{array}\right]
$$

The elements of $\mathbf{Z}(t)$ in (10) are zero-mean, wide-sense stationary, Gaussian random processes. We can express the cross-spectral density matrix of $\mathbf{Z}(t)$ in a convenient form with the following definitions. The array manifold for array $h$ at frequency $\omega$ is

$$
\mathbf{a}_{h}(\omega)=\left[\begin{array}{c}
\exp \left(-j \omega \tau_{h 1}\right) \\
\vdots \\
\exp \left(-j \omega \tau_{h, N_{h}}\right)
\end{array}\right]=\left[\begin{array}{c}
\exp \left[j \frac{\omega}{c}\left(\left(\cos \phi_{h}\right) \Delta x_{h 1}+\left(\sin \phi_{h}\right) \Delta y_{h 1}\right)\right] \\
\vdots \\
\exp \left[j \frac{\omega}{c}\left(\left(\cos \phi_{h}\right) \Delta x_{h, N_{h}}+\left(\sin \phi_{h}\right) \Delta y_{h, N_{h}}\right)\right]
\end{array}\right],
$$

using $\tau_{h n}$ from (2) and assuming that the sensors have omnidirectional response to sources in the plane of interest. Let us define the relative time delay of the signal at arrays $g$ and $h$ as

$$
D_{g h}=\tau_{g}-\tau_{h},
$$

where $\tau_{h}$ is defined in (1). Then the cross-spectral density matrix of $\mathbf{Z}(t)$ in (10) has the form

$$
\begin{aligned}
& \mathbf{G}_{\mathbf{Z}}(\omega)= \\
& {\left[\begin{array}{ccc}
\mathbf{a}_{1}(\omega) \mathbf{a}_{1}(\omega)^{H} G_{s, 11}(\omega) & \cdots & \mathbf{a}_{1}(\omega) \mathbf{a}_{H}(\omega)^{H} \exp \left(-j \omega D_{1 H}\right) G_{s, 1 H}(\omega) \\
\vdots & \ddots & \vdots \\
\mathbf{a}_{H}(\omega) \mathbf{a}_{1}(\omega)^{H} \exp \left(+j \omega D_{1 H}\right) G_{s, 1 H}(\omega)^{*} & \cdots & \mathbf{a}_{H}(\omega) \mathbf{a}_{H}(\omega)^{H} G_{s, H H}(\omega)
\end{array}\right]+G_{w}(\omega) \mathbf{I} .}
\end{aligned}
$$

Recall that the source cross-spectral density functions $G_{s, g h}(\omega)$ in (13) can be expressed in terms of the spectral coherence $\gamma_{s, g h}(\omega)$ using $(7)$.

Note that (13) depends on the source location parameters $\left(x_{s}, y_{s}\right)$ through $\mathbf{a}_{h}(\omega)$ and $D_{g h}$. However, (13) points out that the observations are also characterized by the bearings $\phi_{1}, \ldots, \phi_{H}$ to the source from the individual arrays and the relative time delays $D_{g h}$ between pairs of arrays. Therefore, one way to estimate the source location $\left(x_{s}, y_{s}\right)$ is to estimate the bearings $\phi_{1}, \ldots, \phi_{H}$ and the pairwise time delays $D_{g h}$.

\subsection{Signal coherence in measured data}

Next we present results from measured aeroacoustic data to illustrate typical values of signal coherence at distributed arrays. The experimental setup is illustrated in Figure 2a, which shows the path of a moving ground vehicle and the locations of four microphone arrays (labeled 1, 3, 4,5). Each array is circular with $N=7$ sensors, 4 -ft radius, and six sensors equally spaced around the perimeter with one sensor in the center. We focus on the 10 second segment indicated by the $\diamond$ 's in Figure 2a (which correspond to the time segment 340-350 sec in the data). Figure 2b shows the power spectral density (PSD) of the data measured at arrays 1 and 3 during the 10 second segment. Note the dominant harmonic at $39 \mathrm{~Hz}$. Figure 2c shows the estimated coherence between arrays 1 and 3 during the 10 second segment. The coherence is approximately 0.85 at $40 \mathrm{~Hz}$, which demonstrates the presence of significant coherence at widely-separated microphones. Exploiting this coherence has the potential for improved source localization accuracy. Figure 2 d shows the estimated coherence between two sensors on array 1, spaced by 8 feet. Note that the coherence is close to unity for frequencies in the range from about 40 to $200 \mathrm{~Hz}$, so our model of perfect signal coherence over individual arrays is reasonable.

The Doppler effect due to source motion was compensated prior to the coherence estimate shown in Figure 2c. Without Doppler compensation, the coherence is significantly reduced, as shown in Figure 3a. The time-varying radial velocity of the source with respect to each array in Figure 2a is plotted in the top panel of Figure 3b. If $s(t)$ is 
the waveform emitted by the source that is moving with radial velocity $v$ with respect to the sensor, then the sensor receives a waveform with the form $s(\alpha t)$, where the scaling factor $\alpha$ is

$$
\alpha=1-\frac{v}{c}
$$

and $c$ is the speed of wave propagation. The scaling factor $\alpha$ is plotted in the bottom panel of Figure $3 \mathrm{~b}$. Note that for this data set, $0.98<\alpha<1.02$, which corresponds to a Doppler frequency shift of approximately $\pm 1 \mathrm{~Hz}$ for an emitted tone at 50 hertz. We use a digital resampling algorithm to compensate for the Doppler effect.

\section{CRBS ON LOCALIZATION ACCURACY}

The problem of interest is to estimate the source location parameter vector $\boldsymbol{\Theta}=\left[x_{s}, y_{s}\right]^{T}$ using $T$ samples of the sensor signals $\mathbf{Z}(0), \mathbf{Z}\left(T_{s}\right), \ldots, \mathbf{Z}\left((T-1) \cdot T_{s}\right)$, where $T_{s}$ is the sampling period. Let us denote the sampling rate by $f_{s}=1 / T_{s}$ and $\omega_{s}=2 \pi f_{s}$. We will assume that the continuous-time random processes $\mathbf{Z}(t)$ are band-limited, and that the sampling rate $f_{s}$ is greater than twice the bandwidth of the processes. Then Friedlander ${ }^{19}$ has shown, using a theorem of Whittle, ${ }^{20}$ that the Fisher information matrix (FIM) $\mathbf{J}$ for the parameters $\boldsymbol{\Theta}$ based on the samples $\mathbf{Z}(0), \mathbf{Z}\left(T_{s}\right), \ldots, \mathbf{Z}\left((T-1) \cdot T_{s}\right)$ has elements

$$
J_{i j}=\frac{T}{2 \omega_{s}} \int_{0}^{\omega_{s}} \operatorname{tr}\left\{\frac{\partial \mathbf{G}_{\mathbf{Z}}(\omega)}{\partial \theta_{i}} \mathbf{G}_{\mathbf{Z}}(\omega)^{-1} \frac{\partial \mathbf{G}_{\mathbf{Z}}(\omega)}{\partial \theta_{j}} \mathbf{G}_{\mathbf{Z}}(\omega)^{-1}\right\} d \omega, \quad i, j=1,2
$$

where "tr" denotes the trace of the matrix. The CRB matrix $\mathbf{C}=\mathbf{J}^{-1}$ then has the property that the covariance matrix of any unbiased estimator $\hat{\boldsymbol{\Theta}}$ satisfies $\operatorname{Cov}(\hat{\boldsymbol{\Theta}})-\mathbf{C} \geq \mathbf{0}$, where $\geq \mathbf{0}$ means that $\operatorname{Cov}(\hat{\boldsymbol{\Theta}})-\mathbf{C}$ is positive semidefinite. ${ }^{18}$ The CRB provides a lower bound on the performance of any unbiased estimator. Equation (15) provides a convenient way to compute the FIM for the distributed sensor array model. It provides a powerful tool for evaluating the impact that various parameters have on source localization accuracy. Parameters of interest include the spectral coherence between distributed arrays, the signal bandwidth and power spectrum, the array placement geometry, and the SNR. The FIM in (15) is not easily evaluated analytically, but it is readily evaluated numerically for cases of interest.

Consider an acoustic source that has a narrowband power spectrum. That is, the PSD $G_{s, h h}(\omega)$ of the signal at each array $h=1, \ldots, H$ is nonzero only in a narrow band of frequencies $\omega_{0}-(\Delta \omega / 2) \leq \omega \leq \omega_{0}+(\Delta \omega / 2)$. If the bandwidth $\Delta \omega$ is chosen small enough so that the $\omega$-dependent quantities in (15) are well approximated by their value at $\omega_{0}$, then the narrowband approximation to the FIM (15) is

$$
J_{i j} \approx \frac{T \Delta \omega}{\omega_{s}} \operatorname{tr}\left\{\frac{\partial \mathbf{G}_{\mathbf{Z}}\left(\omega_{0}\right)}{\partial \theta_{i}} \mathbf{G}_{\mathbf{Z}}\left(\omega_{0}\right)^{-1} \frac{\partial \mathbf{G}_{\mathbf{Z}}\left(\omega_{0}\right)}{\partial \theta_{j}} \mathbf{G}_{\mathbf{Z}}\left(\omega_{0}\right)^{-1}\right\}
$$

The quantity $\frac{T \Delta \omega}{\omega_{s}}$ multiplying the FIM in (16) is the time-bandwidth product of the observations. In narrowband array processing, the $T$ time samples per sensor are often segmented into $M$ blocks containing $T / M$ samples each. Then the discrete Fourier transform (DFT) is applied to each block, and the complex coefficients at frequency $\omega_{0}$ (at each sensor) are used to form $M$ array "snapshots". In this case, the quantity $\frac{T \Delta \omega}{\omega_{s}}$ is approximately equal to $M$.

\section{THRESHOLD COHERENCE FOR TIME DELAY ESTIMATION}

In this section, we present a model for partial signal coherence in terms of an equivalent additive noise component, which allows the fundamental bounds on time delay estimation ${ }^{16}$ to be extended to the case of partially coherent signals. Bounds on time delay estimation are important because one of the suboptimum methods for distributed processing with low communication bandwidth ${ }^{1}$ requires time delay estimation between widely separated sensors. The fundamental bounds ${ }^{16}$ are useful because they specify the required signal-to-noise ratio (SNR) such that the CRB is attainable. That is, if the SNR is less than a threshold, then the CRB on source localization accuracy is an optimistic and unattainable lower bound. We will show that for signals that are partially coherent when received at the sensors, a threshold phenomenon occurs with respect to coherence. That is, the signal coherence must exceed a threshold in order for the CRBs to be attainable. The existence of a threshold phenomenon for signal coherence is a useful refinement of the CRB results, ${ }^{1}$ since the $\mathrm{CRBs}^{1}$ are formulated without regard for the conditions of attainability. 


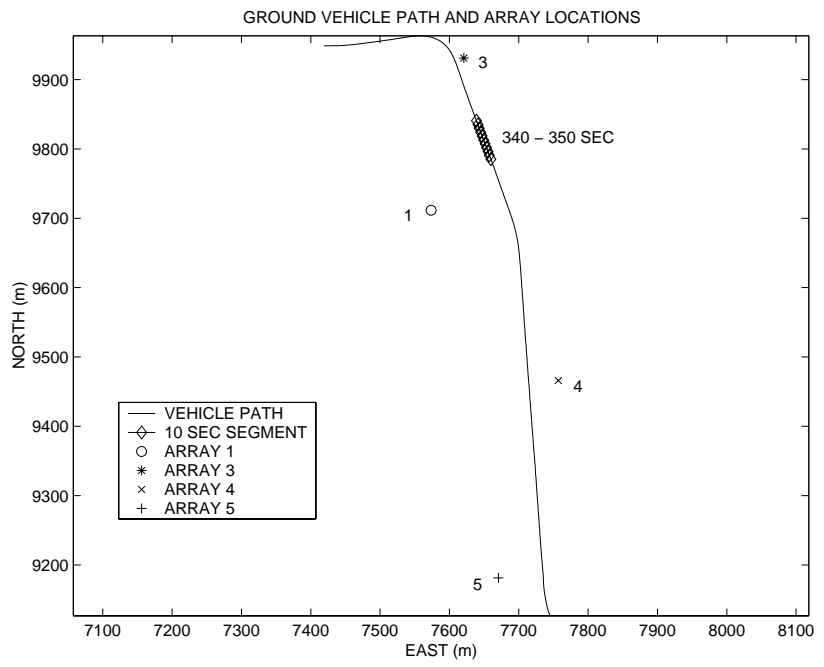

(a)

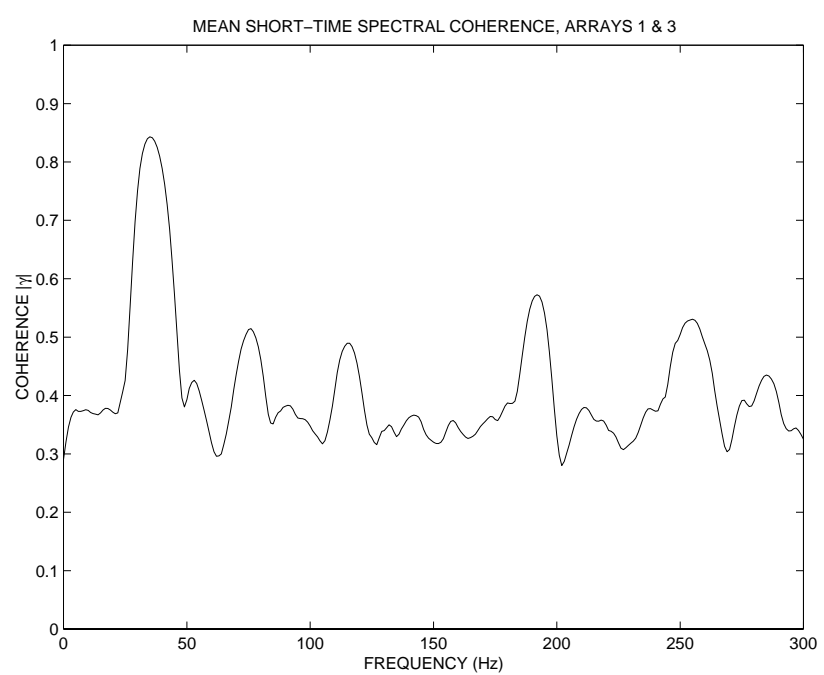

(c)
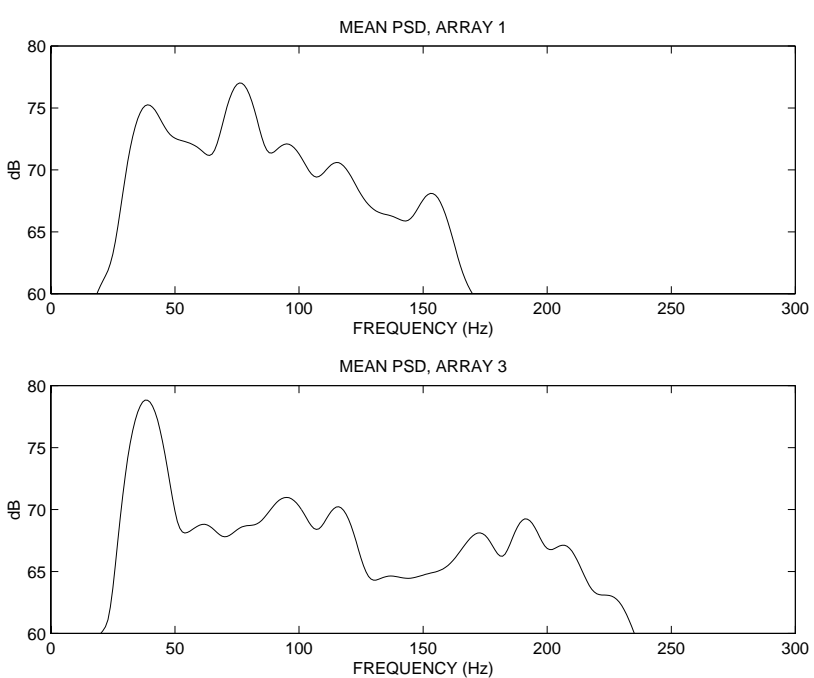

(b)

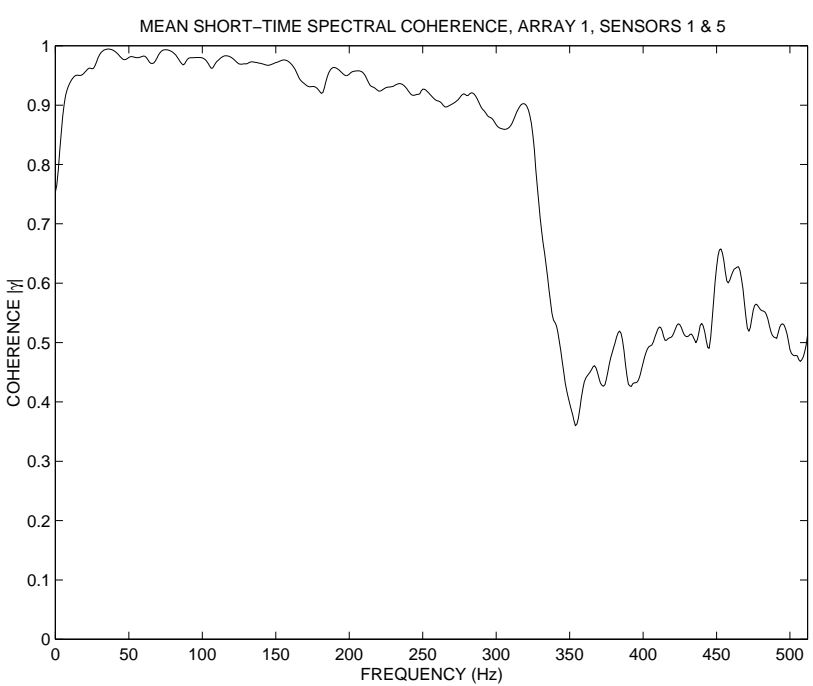

(d)

Figure 2. (a) Path of ground vehicle and array locations for measured data. (b) Mean power spectral density (PSD) at arrays 1 and 3 estimated from measured data over the 10 second segment $\diamond$ in (b). Top panel is $G_{s, 11}(f)$, bottom panel is $G_{s, 33}(f)$. (c) Mean spectral coherence $\gamma_{s, 13}(f)$ between arrays 1 and 3 estimated over the 10 second segment. (d) Mean spectral coherence for two sensors on array 1, with sensor spacing 8 feet. 


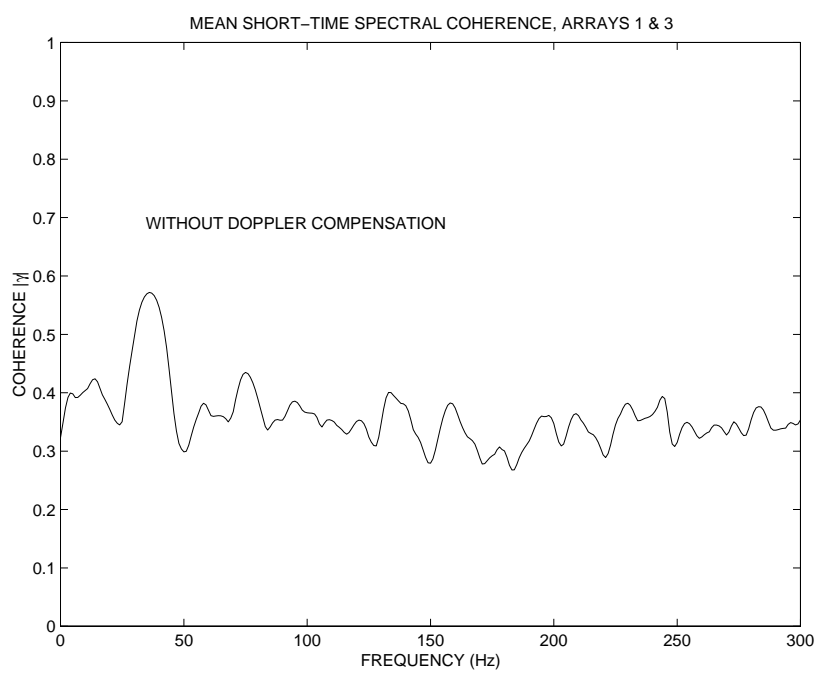

(a)
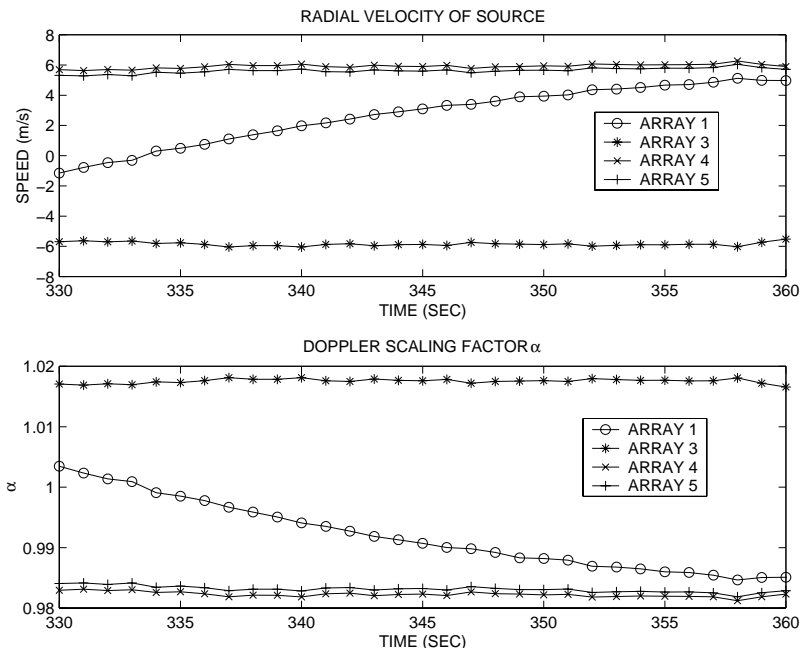

(b)

Figure 3. (a) Mean short-time spectral coherence between arrays 1 and 3 if Doppler is not compensated. (b) Radial velocity and Doppler scaling factor $\alpha$ in (14) for source in Figure 2a with respect to each array.

The appendix presents a decomposition of partially coherent signals into a coherent component and an incoherent additive noise component. Consider estimation of the time delay $D$ in the model presented as (35),(36) and (45),(46) in the appendix. Let us specialize to narrowband processing, with signal bandwidth $\Delta \omega$ centered at $\omega_{0}$ and observation time $T$ seconds. Further, we assume that the signal power is identical at each sensor, and we define the following constants for notational simplicity:

$$
G_{s, 11}\left(\omega_{0}\right)=G_{s, 2}\left(\omega_{0}\right)=G_{s}, \quad G_{w}\left(\omega_{0}\right)=G_{w}, \quad \gamma_{s, 12}\left(\omega_{0}\right)=\gamma_{s} .
$$

Then combining the formulation in the appendix with previously developed Ziv-Zakai bounds ${ }^{16}$ we can show that the following SNR expression characterizes the performance of time delay estimation with partially coherent signals:

$$
\operatorname{SNR}\left(\gamma_{s}\right)=\left[\frac{1}{\left|\gamma_{s}\right|^{2}}\left(1+\frac{1}{\left(G_{s} / G_{w}\right)}\right)^{2}-1\right]^{-1} .
$$

The threshold SNR for CRB attainability in the narrowband time delay estimation problem is ${ }^{16}$

$$
\mathrm{SNR}_{\text {thresh }}=\frac{6}{\pi^{2}\left(\frac{\Delta \omega T}{2 \pi}\right)}\left(\frac{\omega_{0}}{\Delta \omega}\right)^{2}\left[\phi^{-1}\left(\frac{(\Delta \omega)^{2}}{24 \omega_{0}^{2}}\right)\right]^{2}
$$

where $\phi(y)=1 / \sqrt{2 \pi} \int_{y}^{\infty} \exp \left(-t^{2} / 2\right) d t$. Thus

$$
\operatorname{SNR}\left(\gamma_{s}\right) \geq \operatorname{SNR}_{\text {thresh }}
$$

identifies the values of signal coherence $\gamma_{s}$ and signal/noise PSDs $G_{s} / G_{w}$ for CRB attainability. We can combine (18) with (20) to obtain the condition for CRB attainability

$$
\frac{G_{s}}{G_{w}} \geq \frac{1}{\left|\gamma_{s}\right|\left(1+\frac{1}{\text { SNR }_{\text {thresh }}}\right)^{1 / 2}-1},
$$




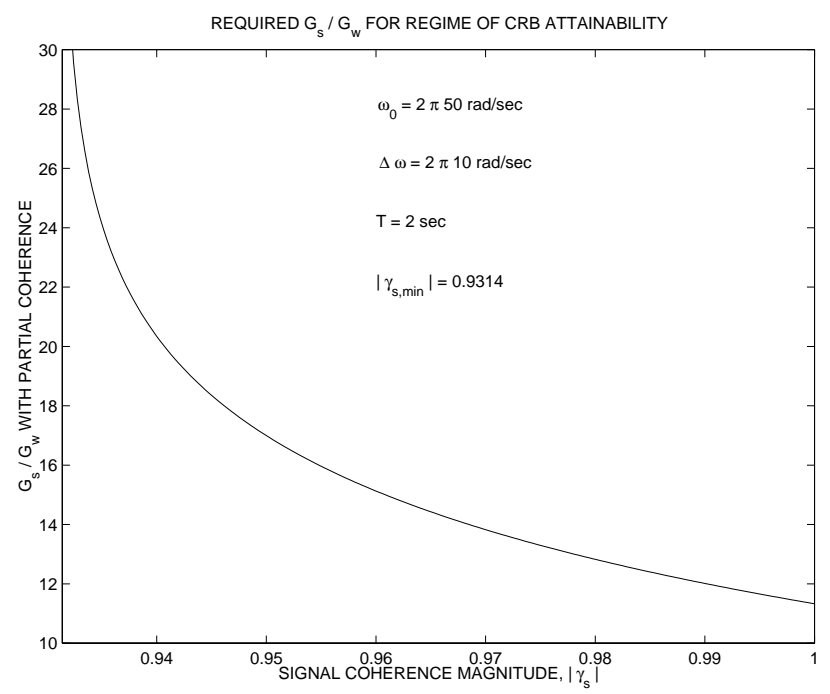

(a)

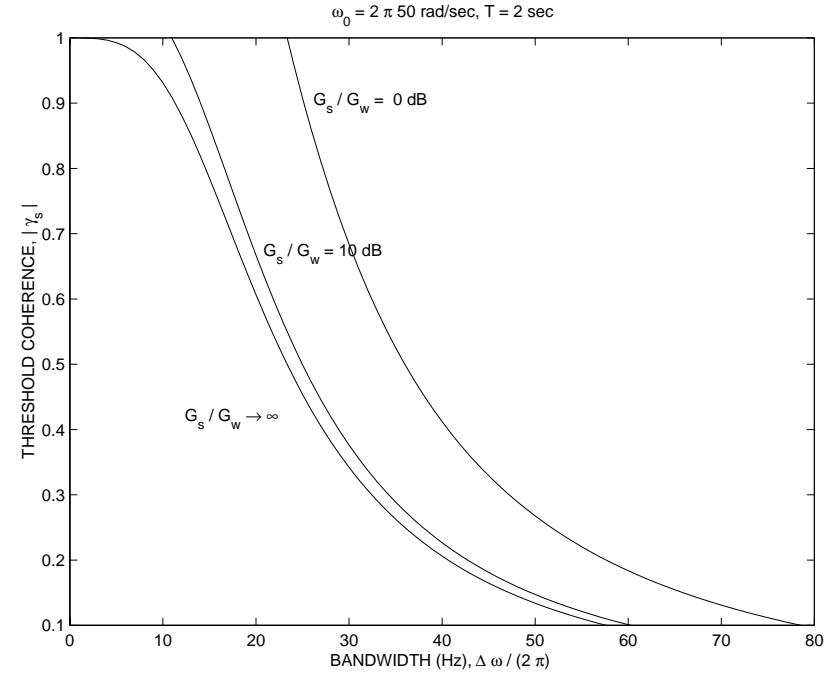

(b)

Figure 4. (a) Required signal to noise ratio $G_{s} / G_{w}$ versus coherence $\gamma_{s}$, based on (21) for $\omega_{0}=2 \pi 50 \mathrm{rad} / \mathrm{sec}$, $\Delta \omega=2 \pi 10 \mathrm{rad} / \mathrm{sec}, T=2 \mathrm{sec}$. (b) Threshold coherence versus bandwidth based on (22) for $\omega_{0}=2 \pi 50 \mathrm{rad} / \mathrm{sec}$ and $G_{s} / G_{w}=0,10$, and $\infty \mathrm{dB}$.

which is possible only if

$$
\left|\gamma_{s}\right|^{2} \geq \frac{\left(1+\frac{1}{\left(G_{s} / G_{w}\right)}\right)^{2}}{1+\frac{1}{\mathrm{SNR}_{\text {thresh }}}}, \quad \text { where }\left|\gamma_{s}\right|^{2} \geq \frac{1}{1+\frac{1}{\mathrm{SNR}_{\text {thresh }}}} \text { as } \frac{G_{s}}{G_{w}} \rightarrow \infty .
$$

For a specific narrowband time delay estimation scenario, the threshold SNR for CRB attainability is given by (19), and (22) provides a corresponding threshold coherence for CRB attainability.

Figure 4 contains plots of (21) and (22) for time delay estimation with signals in a band centered at $\omega_{0}=2 \pi 50$ $\mathrm{rad} / \mathrm{sec}$ using a time segment of duration $T=2$ seconds. Note in Figure 4a that the threshold coherence value is 0.9314 for $G_{s} / G_{w} \rightarrow \infty$, and that larger coherence is required for finite values of $G_{s} / G_{w}$. Figure $4 \mathrm{~b}$ shows the variation in threshold coherence as a function of signal bandwidth, $\Delta \omega$. Note that nearly perfect coherence is required when the signal bandwidth is less than $5 \mathrm{~Hz}$ (or 10\% fractional bandwidth). The threshold coherence drops sharply for values of signal bandwidth greater than $10 \mathrm{~Hz}$ (20\% fractional bandwidth). Thus for sufficiently wideband signals, e.g., $\Delta \omega \geq 2 \pi 10 \mathrm{rad} / \mathrm{sec}$, a certain amount of coherence loss can be tolerated while still allowing unambiguous time delay estimation.

\subsection{Examples}

First we present an illustration based on processing the measured data for the source in Figure 2a. Figure 5 shows results of cross-correlation processing of the data for a 2 second segment at time 342 seconds. Figure 5 a is obtained by cross-correlating the signals received at arrays 1 and 3, for which the coherence is appreciable only over a narrow band near $39 \mathrm{~Hz}$. A peak in the cross-correlation is not evident, which is expected based on the preceding analysis, since nearly perfect coherence is needed for narrowband time delay estimation in this scenario. Figure 5b is obtained by cross-correlating the signals received at two sensors on array 1, where the coherence is as shown in Figure $2 \mathrm{~d}$. Due to the high signal coherence over a wide band, a peak is clearly evident in the cross-correlation.

Next we present an illustration based on measured data with a synthetically-generated, non-moving, wideband acoustic source. The location of the source and two sensors ( 0 and 1$)$ form a triangle as follows: the distance from the 

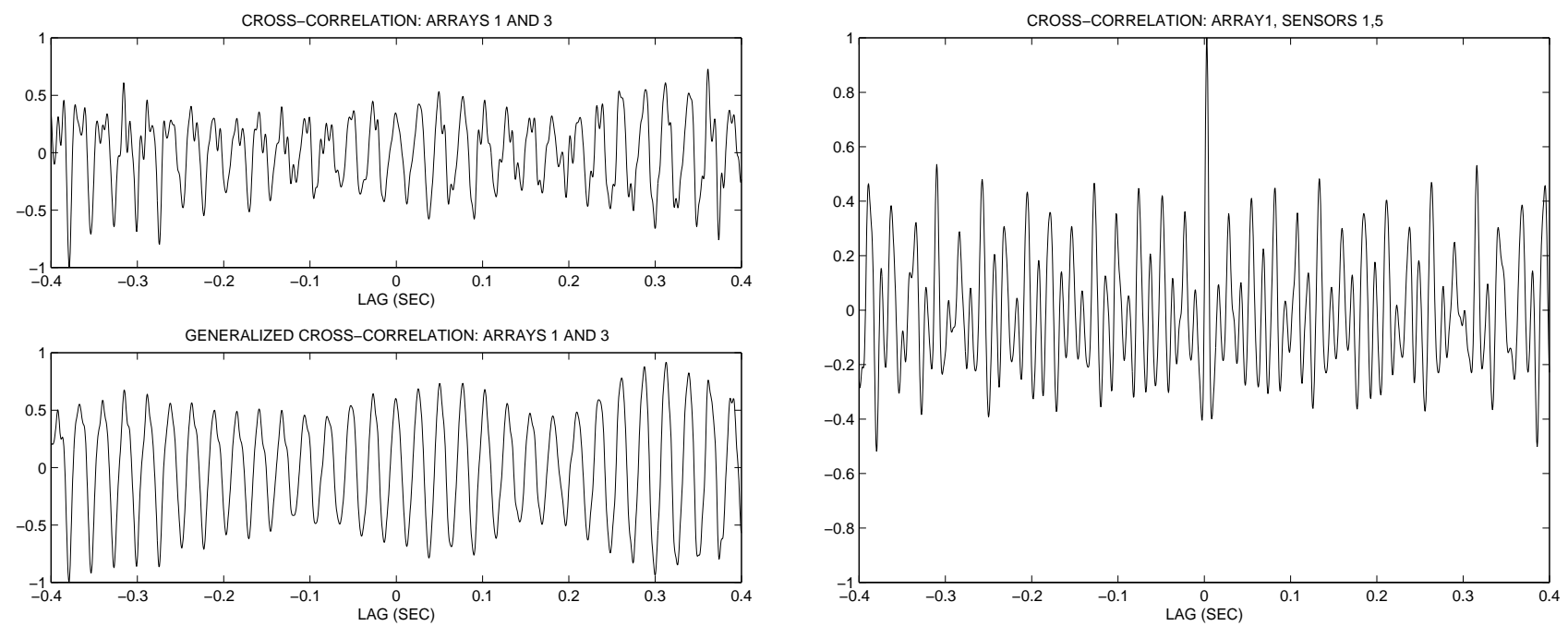

(a)

(b)

Figure 5. (a) Cross-correlation of signals at arrays 1 and 3 in Figure 2a for source at time 342 sec. (b) Crosscorrelation of signals at two sensors on array 1, separated by 8 feet.

source to sensors 0 and 1 is 233 feet and 329 feet, respectively, and the distance between sensors 0 and 1 is 233 feet. The power spectral density (PSD) and coherence magnitude estimated from 1-second segments of data measured at sensors 0 and 1 is shown in Figure 6a. The actual transmitted signal has a bandwidth of approximately $50 \mathrm{~Hz}$ that is centered at $100 \mathrm{~Hz}$. Note that the PSDs of the sensor signals do not have their maxima at $100 \mathrm{~Hz}$ due to the acoustic propagation. However, the coherence magnitude is roughly 0.8 over a $50 \mathrm{~Hz}$ band centered at $100 \mathrm{~Hz}$. The threshold coherence is exceeded for this data set, so TDE between sensors 0 and 1 should be feasible. Figure $6 \mathrm{~b}$ shows the generalized cross-correlation with peak at zero lag, which is the correct location because the sensor data is time-aligned. This example shows the feasibility of TDE with acoustic signals measured at widely-separated sensors, provided that the SNR, fractional bandwidth, time-bandwidth product, and coherence are such that the thresholds illustrated in Figure 4 are exceeded.

\section{CONCLUDING REMARKS}

Items of continuing work include the following. We are continuing to process measured data with moving ground vehicles that exhibit coherence and bandwidth levels that exceed the thresholds presented in this paper. Our objective is to demonstrate the improved source localization accuracy when TDEs between distributed arrays is processed with the bearing estimates from individual arrays.

The model in this paper assumes that the source location is fixed over the observation time. We are investigating models for the source motion that will improve the accuracy of source localization, Doppler correction, and tracking. Also, we are investigating extensions to the case of tracking multiple moving sources.

\section{APPENDIX A. EQUIVALENT ADDITIVE NOISE MODEL FOR COHERENCE}

The formulation in this appendix begins with Gaussian random variables for simplicity, and then the formulation is extended to Gaussian random processes.

Let $X$ and $Y$ be two complex, circular, Gaussian random variables with zero mean and

$$
E\left\{|X|^{2}\right\}=\sigma_{X}^{2}, E\left\{|Y|^{2}\right\}=\sigma_{Y}^{2}, E\left\{X Y^{*}\right\}=\rho \sigma_{X} \sigma_{Y} .
$$



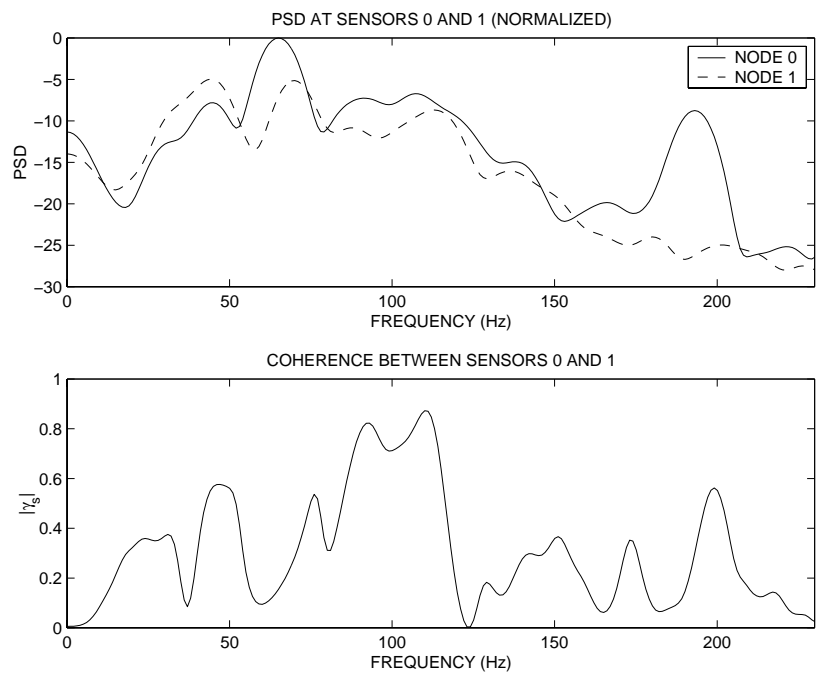

(a)

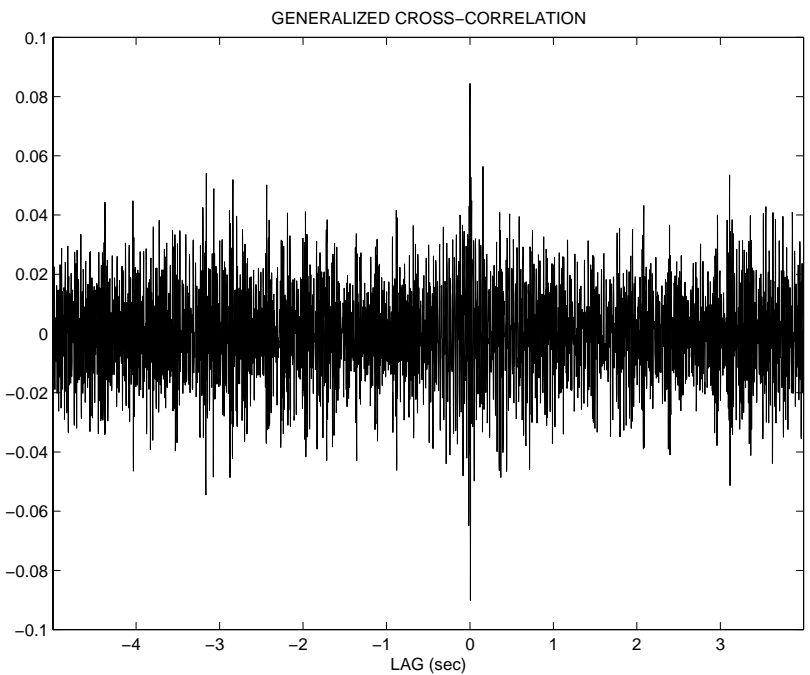

(b)

Figure 6. (a) Power spectral density (PSD) and coherence for synthetically-generated wideband source located 233 feet from sensors 0 and 1. (b) Generalized cross-correlation of 1-second segment from one sensor with 10-second segment from other sensor, with time-alignment so the peak is ideally at zero lag.

Then $X$ and $Y$ can be expressed as

$$
\begin{aligned}
& X=\sigma_{X} S+N_{1} \\
& Y=\frac{\rho^{*}}{|\rho|} \sigma_{Y} S+N_{2},
\end{aligned}
$$

where $S, N_{1}, N_{2}$ are independent, zero mean, complex, circular Gaussian random variables with

$$
\begin{aligned}
E\left\{|S|^{2}\right\} & =|\rho| \\
E\left\{\left|N_{1}\right|^{2}\right\} & =\sigma_{X}^{2}(1-|\rho|) \\
E\left\{\left|N_{2}\right|^{2}\right\} & =\sigma_{Y}^{2}(1-|\rho|) .
\end{aligned}
$$

The relations in (23) can be verified using (24)-(28). Note that for $X$, the "coherent" part $\sigma_{X} S$ has variance $|\rho| \sigma_{X}^{2}$, while the "incoherent" part $N_{1}$ has variance $(1-|\rho|) \sigma_{X}^{2}$. As $|\rho|$ decreases, a larger portion of $\sigma_{X}^{2}$ is applied to $N_{1}$, so the incoherent noise gets stronger.

Suppose that $X$ and $Y$ described by (23) are observed in noise,

$$
\begin{aligned}
& Z_{1}=X+W_{1}=\sigma_{X} S+N_{1}+W_{1} \\
& Z_{2}=Y+W_{1}=\frac{\rho^{*}}{|\rho|} \sigma_{Y} S+N_{2}+W_{2},
\end{aligned}
$$

where $W_{1}$ and $W_{2}$ are independent, zero mean, complex, circular Gaussian random variables with variance $\sigma_{W}^{2}$, and $W_{1}, W_{2}$ are independent of $X, Y$ (and therefore $S, N_{1}, N_{2}$ ). If we define the SNR of $Z_{1}$ and $Z_{2}$ based only on the coherent signal component, then

$$
\mathrm{SNR}_{1}=\frac{|\rho| \sigma_{X}^{2}}{\sigma_{W}^{2}+(1-|\rho|) \sigma_{X}^{2}}=\frac{|\rho|}{\frac{\sigma_{W}^{2}}{\sigma_{X}^{2}}+(1-|\rho|)}
$$




$$
\mathrm{SNR}_{2}=\frac{|\rho| \sigma_{Y}^{2}}{\sigma_{W}^{2}+(1-|\rho|) \sigma_{Y}^{2}}=\frac{|\rho|}{\frac{\sigma_{W}^{2}}{\sigma_{Y}^{2}}+(1-|\rho|)} .
$$

Some observations about (31) and (32) follow.

1. $\mathrm{SNR}_{1}$ and $\mathrm{SNR}_{2}$ increase monotonically as $|\rho|$ increases.

2. $\mathrm{SNR}_{1}\left(\mathrm{SNR}_{2}\right)$ increases monotonically as $\sigma_{X}^{2}\left(\sigma_{Y}^{2}\right)$ increases, and for fixed $|\rho|$ is bounded by

$$
\lim _{\sigma_{X}^{2} \rightarrow \infty} \mathrm{SNR}_{1}=\lim _{\sigma_{Y}^{2} \rightarrow \infty} \mathrm{SNR}_{2}=\frac{|\rho|}{1-|\rho|} .
$$

Thus a loud source (large $\sigma_{X}^{2}, \sigma_{Y}^{2}$ ) has larger coherent SNR, i.e., increasing $\sigma_{X}^{2}, \sigma_{Y}^{2}$ cannot reduce the coherent SNR, but the limit (33) cannot be exceeded for a given $|\rho|$.

3. If a particular coherent SNR is desired, then (33) implies a threshold coherence magnitude $|\rho|$ that is required to achieve that SNR, given by

$$
|\rho| \geq \frac{\mathrm{SNR}}{1+\mathrm{SNR}}
$$

Note that for large coherent SNR, nearly perfect coherence $|\rho| \approx 1$ is required.

The analysis extends to complex Gaussian random processes as follows. Consider a time delay estimation problem with two sensors and noisy observations of the form in (8),

$$
\begin{aligned}
& z_{1}(t)=s_{1}(t)+w_{1}(t) \\
& z_{2}(t)=s_{2}(t-D)+w_{2}(t),
\end{aligned}
$$

where $w_{1}(t), w_{2}(t)$ are additive, white, Gaussian noise (AWGN) processes, $s_{1}(t), s_{2}(t)$ are partially coherent Gaussian random processes, and $D$ is the time delay. The AWGN processes $w_{1}(t), w_{2}(t)$ have properties as in (9) with power spectral density (PSD) $G_{w}(\omega)$, and the signals $s_{1}(t), s_{2}(t)$ are characterized by the cross-spectral density matrix

$$
\left[\begin{array}{c}
s_{1}(t) \\
s_{2}(t)
\end{array}\right] \sim\left[\begin{array}{cc}
G_{s, 11}(\omega) & \gamma_{s, 12}(\omega)\left(G_{s, 11}(\omega) G_{s, 22}(\omega)\right)^{1 / 2} \\
\gamma_{s, 12}(\omega)^{*}\left(G_{s, 11}(\omega) G_{s, 22}(\omega)\right)^{1 / 2} & G_{s, 22}(\omega)
\end{array}\right] .
$$

Then analogous to $(24),(25), s_{1}(t)$ and $s_{2}(t)$ can be represented as

$$
\begin{aligned}
& s_{1}(t)=h_{1}(t) * s(t)+n_{1}(t) \\
& s_{2}(t)=h_{2}(t) * s(t)+n_{2}(t)
\end{aligned}
$$

where $s(t), n_{1}(t), n_{2}(t)$ are independent, zero mean, circular, complex Gaussian random processes, $*$ denotes convolution, and

$$
\begin{aligned}
H_{1}(\omega) & =G_{s, 11}(\omega)^{1 / 2} \\
H_{2}(\omega) & =\frac{\gamma_{s, 12}(\omega)^{*}}{\left|\gamma_{s, 12}(\omega)\right|} G_{s, 22}(\omega)^{1 / 2} \\
G_{s}(\omega) & =\left|\gamma_{s, 12}(\omega)\right| \\
G_{1}(\omega) & =G_{s, 11}(\omega)\left[1-\left|\gamma_{s, 12}(\omega)\right|\right] \\
G_{2}(\omega) & =G_{s, 22}(\omega)\left[1-\left|\gamma_{s, 12}(\omega)\right|\right] .
\end{aligned}
$$

$G_{s}(\omega), G_{1}(\omega), G_{2}(\omega)$ are the PSDs of $s(t), n_{1}(t), n_{2}(t)$, and $H_{1}(\omega), H_{2}(\omega)$ are the frequency responses corresponding to $h_{1}(t), h_{2}(t)$ that model the deterministic "channels" from source to sensors. Then (38),(39) can be inserted into $(35),(36)$,

$$
\begin{aligned}
& z_{1}(t)=\left(h_{1} * s\right)(t)+n_{1}(t)+w_{1}(t) \\
& z_{2}(t)=\left(h_{2} * s\right)(t-D)+n_{2}(t)+w_{2}(t)
\end{aligned}
$$


which fits the standard model for time delay estimation of coherent signals observed through linear filters in AWGN. ${ }^{21}$ The partial signal coherence $\gamma_{s, 12}(\omega)$ between $s_{1}(t), s_{2}(t)$ in $(35),(36)$ is equivalently modeled by the filtered coherent signals $\left(h_{1} * s\right),\left(h_{2} * s\right)$ and the excess additive noise $n_{1}(t), n_{2}(t)$.

\section{REFERENCES}

1. R.J. Kozick and B.M. Sadler, "Distributed sensor array processing of wideband acoustic signals," IRIS Battlefield Acoustics Symposium, September 1999.

2. R.J. Kozick and B.M. Sadler, "Algorithms for Localization and Tracking of Acoustic Sources with Widely Separated Sensors," Proc. 2000 Meeting of the IRIS Specialty Group on Battlefield Acoustics and Seismics, Laurel, MD, October 18-20, 2000.

3. D.K. Wilson, "Atmospheric effects on acoustic arrays: a broad perspective from models," 1999 Meeting of the IRIS Specialty Group on Battlefield Acoustics and Seismics, Laurel, MD, September 13-15, 1999.

4. T. Pham and B. M. Sadler, "Aeroacoustic wideband array processing for detection and tracking of ground vehicles," 130th Meeting of the Acoustic Society of America, St. Louis, MI, JASA vol. 98, no. 5, pt. 2, p. 2969, 1995.

5. T. Pham and B. M. Sadler, "Adaptive wideband aeroacoustic array processing," 8th IEEE Statistical Signal and Array Processing Workshop, pp. 295-298, Corfu, Greece, June 1996.

6. T. Pham and B. Sadler, "Incoherent and coherent wideband direction finding algorithms for ground vehicles," 132nd Meeting of the Acoustic Society of America, JASA vol. 100, no. 4, pt. 2, p. 2636, October 1996.

7. T. Pham and B. M. Sadler, "Focused wideband array processing algorithms for high-resolution direction finding," IRIS Battlefield Acoustics Symposium, October 1998.

8. H. Wang and M. Kaveh, "Coherent signal-subspace processing for the detection and estimation of angles of arrival of multiple wide-band sources," IEEE Trans. Acoust., Speech, Signal Processing, vol. ASSP-33, pp. 823-831, August 1985.

9. B. M. Sadler, "Focused wideband maximum likelihood and spatial spectrum estimation" 920th Meeting of the American Mathematical Society, invited presentation for Special Session on Harmonic Analysis and Applications, College Park, MD, April 1997.

10. A. Paulraj and T. Kailath, "Direction of arrival estimation by eigenstructure methods with imperfect spatial coherence of wavefronts," J. Acoust. Soc. Am., vol. 83, pp. 1034-1040, March 1988.

11. A.B. Gershman, C.F. Mecklenbrauker, J.F. Bohme, "Matrix fitting approach to direction of arrival estimation with imperfect spatial coherence," IEEE Trans. on Signal Proc., vol. 45, no. 7, pp. 1894-1899, July 1997.

12. B.-G. Song and J.A. Ritcey, "Angle of arrival estimation of plane waves propagating in random media," $J$. Acoust. Soc. Am., vol. 99, no. 3, pp. 1370-1379, March 1996.

13. D.K. Wilson, "Performance bounds for acoustic direction-of-arrival arrays operating in atmospheric turbulence," J. Acoust. Soc. Am., vol. 103, no. 3, pp. 1306-1319, March 1998.

14. M. Wax and T. Kailath, "Decentralized processing in sensor arrays," IEEE Trans. on Acoustics, Speech, Signal Processing, vol. ASSP-33, no. 4, pp. 1123-1129, October 1985.

15. E. Weinstein, "Decentralization of the Gaussian maximum likelihood estimator and its applications to passive array processing," IEEE Trans. Acoust., Speech, Sig. Proc., vol. ASSP-29, no. 5, pp. 945-951, October 1981.

16. A.J. Weiss and E. Weinstein, "Fundamental limitations in passive time delay estimation - part 1: narrowband systems," IEEE Trans. Acoust., Speech, Sig. Proc., vol. ASSP-31, no. 2, pp. 472-485, April 1983.

17. D.K. Wilson, G.L. Szeto, B.M. Sadler, R. Adams, N. Srour, "Propagation and array performance modeling for acoustic tracking of cruise missiles," 1999 Meeting of the IRIS Specialty Group on Battlefield Acoustics and Seismics, Laurel, MD, September 13-15, 1999.

18. S.M. Kay, Fundamentals of Statistical Signal Processing: Estimation Theory, Prentice-Hall, 1993.

19. B. Friedlander, "On the Cramer-Rao Bound for Time Delay and Doppler Estimation," IEEE Trans. on Info. Theory, vol. IT-30, no. 3, pp. 575-580, May 1984.

20. P. Whittle, "The analysis of multiple stationary time series," J. Royal Statist. Soc., vol. 15, pp. 125-139, 1953.

21. G.C. Carter (ed.), Coherence and Time Delay Estimation (Selected Reprint Volume), IEEE Press, 1993.

22. C.H. Knapp and G.C. Carter, "The generalized correlation method for estimation of time delay," IEEE Trans. on Acoustics, Speech, Signal Processing, vol. ASSP-24, no. 4, pp. 320-327, August 1976. 